\title{
Formalization and the Objects of Logic
}

\section{Journal Article}

Author(s):

Brun, Georg

Publication date:

2008

Permanent link:

https://doi.org/10.3929/ethz-b-000013509

Rights / license:

In Copyright - Non-Commercial Use Permitted

Originally published in:

Erkenntnis 69(1), https://doi.org/10.1007/s10670-008-9112-3 


\title{
Formalization and the Objects of Logic
}

\author{
Georg Brun
}

Received: 1 March 2007/Accepted: 9 April 2008/Published online: 14 May 2008

(C) Springer Science+Business Media B.V. 2008

\begin{abstract}
There is a long-standing debate whether propositions, sentences, statements or utterances provide an answer to the question of what objects logical formulas stand for. Based on the traditional understanding of logic as a science of valid arguments, this question is firstly framed more exactly, making explicit that it calls not only for identifying some class of objects, but also for explaining their relationship to ordinary language utterances. It is then argued that there are strong arguments against the proposals commonly put forward in the debate. The core of the problem is that an informative account of the objects formulas stand for presupposes a theory of formalization; that is, a theory that explains what formulas may adequately substitute for an inference in proofs of validity. Although such theories are still subject to research, some consequences can be drawn from an analysis of the reasons why the common accounts featuring sentences, propositions or utterances fail. Theories of formalization cannot refer to utterances qua expressions of propositions; instead they may refer to sentences and rely on additional information about linguistic structure and pragmatic context.
\end{abstract}

Keywords Logic · Philosophy of logic · Formalization · Proposition · Natural language

In the philosophical tradition of logic, the question "What are the objects of logic?" seems to admit of at least one undisputed answer. As Peirce (1902, pp. 20-21) noted more than a 100 years ago: "Nearly a hundred definitions of [logic] have been given. It will, however, generally be conceded that its central problem is the classification of arguments, so that all of those that are bad are thrown into one

G. Brun $(\bowtie)$

Institute for Environmental Decisions, ETH Zürich, CHN H 73.2, Universitaetstrasse 16, 8092 Zürich, Switzerland

e-mail: Georg.Brun@env.ethz.ch 
division, and those which are good into another." But as soon as we want a more specific answer than "arguments", disagreement starts. To be sure, arguments consist of premises and conclusions, but as what kind of objects should we think of these? Some combinations of premises and conclusion constitute valid inferences, but what is it that stands in this relation of valid inference? At this point we face a well-known debate between proponents of either propositions, sentences, statements or utterances. It has led to a situation in which one can seemingly embrace any of these standard answers and be sure that one's opponents face as many objections as one does. This, of course, raises doubts whether the different positions really deal with the same problem. It is also a motivation for re-evaluating some of the arguments in light of a more clearly framed question. Some 10 years ago, Grandy (1993) suggested that we interpret the question "What are the objects of logic?" as equivalent to "What do 'Q' and 'R' stand for?" (using "Q" and "R" as examples of the so-called "propositional variables"). He then argued that there are convincing arguments against the popular proposals for answering that latter question. The core of his criticism is that not enough attention is paid to the relation between logic and ordinary language. This paper further explores this line of thought, based on an explication of Grandy's question.

For the philosophy of logic, the debate about propositions, sentences and so on is a key issue since it concerns the question of how we may understand the common practice of using logical formalisms in philosophy. The assumption that proofs of validity and logical analysis of various expressions can tell us anything about arguments and statements in ordinary language calls for a theory about the relation of logical formulas to premises and conclusions. In a wider context, the question of what objects logical formulas stand for is of interest because it relates to different yet closely connected issues not dealt with in this paper, such as questions about the objects of propositional attitudes and about the bearers of truth and modality.

In Sect. 1, I will first explain how taking seriously the tradition characterized by Peirce motivates Grandy's interpretation of the question about the objects of logic. Subsequently, a more precise formulation of the leading question will be developed. Section 2 will deal with the pros and cons of the most common answers, as well as with conditions any defensible proposal must meet. This discussion will also confront us with a deficit in the logical tradition: the established practice of proving arguments to be valid is not based on a theoretically respectable account of the relation between formulas and the ordinary language expressions they substitute for. Since an extensive treatment of formalization lies outside the scope of this paper, Sect. 3 will discuss only some consequences for theories of formalization which can be drawn from the arguments in Sect. 2.

\section{Explicating the Question}

This section proposes a more exact replacement for the rather vague question about the objects of logic. Firstly, I will outline how a traditional understanding of logic gives us some motivation for focusing on the more specific problem of "What do logical formulas stand for?" I then propose an analysis of this question that relies on 
distinguishing and explicating two uses of the metaphorical "standing for" while still leaving room for the well-known answers "propositions", "sentences" and so on.

In philosophy, logic has traditionally been understood as a theory of valid arguments, hence historic labels such as ars iudicandi or canon of valid reasoning. Importantly, this is taken to cover arguments which are presented in an ordinary language, be that a natural language, such as Greek or English, or a more specialized and regimented idiom of science. Such an understanding does not necessarily imply that logic exclusively deals with valid arguments, but only that this is the "core business" of logic. Theories or formalisms which do not tell us anything about the validity of arguments are not logical theories or logical formalisms. Of course, in philosophy the analysis not of arguments but of particular sentences-or rather certain classes of sentences (or statements, propositions and so on) - plays an important role. But even when logical analysis is done without aiming at proving the validity of any particular argument, qua logical analysis this business can always be understood as an analysis of a sentence with respect to its possible occurrence in (in)valid arguments (Frege 1879, p. 3). On the other hand, the development of mathematical and formalist approaches to logic has established another use of the word "logic" that refers to a purely formal science or a branch of mathematics studying not arguments but certain formal or mathematical objects. From this perspective, investigating the validity of arguments is a task not of "pure" but of "applied" logic. In this paper, I will not rely on such a distinction, but assume the traditional understanding of logic as the study of valid arguments.

This point of view can be elaborated by looking more closely at the established procedure for proving the validity of an argument. Such proofs can be reconstructed as involving the following steps (cf. Rosenberg 1986, pp. 69-71):

0 . If needed, the argument is brought into a standard form, which is a sequence ${ }^{1}$ of premises and a conclusion. Let us call such a sequence an inference. Identifying premises and conclusion can involve a great deal of interpretation and mostly relies not on logic but on techniques of text-analysis. Logical analysis proper starts with inferences and consists of the next three steps. (I will briefly comment on argument-analysis in Sect. 3.2.)

1. The premises and the conclusion are formalized in some logical system $;^{2}$ that is, they each are assigned a (well-formed) formula. Of course, formalizing cannot be reduced to coming up with just any formula, but requires a formula that actually represents a logical form of the premise or conclusion which it is intended to be a formalization of. It often goes unnoticed that this presupposes that there are criteria of adequacy for formalizations. I will come back to this point below.

\footnotetext{
1 Treating inferences as sequences instead of pairs consisting of a set of premises and a conclusion is more convenient for present purposes because it enables us to easily associate formulas with premises.

${ }^{2}$ I will use standard zero- and first-order logic as examples of logical formalisms since they are paradigmatic for the philosophical tradition I am referring to. However, what follows can be applied to wide range of non-standard logics.
} 
2. The syntactical or semantical resources of the logical formalism are used to give a proof which shows that the conclusion-formula is a valid consequence of the premise-formulas.

3. The inference in question is judged to be valid by appeal to the proof given in the preceding step. (If needed, this judgement can then be carried over to the original argument.)

Against the background of this procedure, the question about the objects of logic can now be framed more precisely. To begin with, we can think of proving validity as a practical business and ask as what kind of objects premises and conclusions enter this activity. If we leave aside the possibility that one may silently present inferences to oneself, we can resolve this question by pointing out that inferences can be given only as sequences of utterances, that is, sentence-tokens of some physical make-up (since nothing depends on the medium, I do not distinguish between utterances, inscriptions, digital patterns and so on). ${ }^{3}$ So utterances are the objects one has to deal with when one goes about proving an inference. But in the procedure described above, the utterances presenting the inference are present only at the very beginning when they are to be replaced (in step 1) by formulas which are then used as their proxies, so to speak, throughout the formal proof (step 2). From this point of view, we can therefore say that formulas substitute for utterances. And we may note that any answer to the question "What do formulas stand for?" which does not refer to utterances will be incomplete.

But this should not be taken to imply that proofs of validity treat premises and conclusions as utterances. In fact, as we shall see soon, it would clearly be mistaken to think so. However, if premises and conclusions are not treated as utterances, as what are they treated then? In Grandy's words: "What do 'Q' and 'R' stand for?" Now, since formulas substitute for utterances in formal proofs, whatever about the original utterances is relevant to such a proof must be reflected in the formulas which are used in their place. We can therefore say that in logical proofs the utterances which present the argument are treated as the kind of object formulas stand for. This is the point where the debate about propositions, statements and so on enters the scene. The positions staked out in this dispute can be understood in the following way: claiming that the objects of logic are, for example, propositions amounts to claiming that the utterances which present an argument are relevant to a logical proof only insofar as they express certain propositions; in short, formulas stand for propositions. Given such a position, one can still claim that formulas substitute for utterances, namely those utterances which are suitably related to some object the formula stands for. For the sake of clarity I do not use "stand for" but introduce "substitute for" for this relation between formulas and utterances. ${ }^{4}$

\footnotetext{
${ }^{3}$ Treating arguments in a publicly accessible medium as paradigm cases is characteristic for most traditions of logic in philosophy, but it has not been uncontested. The main alternative consists in giving theoretical primacy to mental states or acts, as can be found in some approaches which give judgements a central role in logic (cf. Martin-Löf 1996) or take Chomsky's theories of language as their starting point (cf. Collins 2003). Insofar as mental states or acts are singular events or objects, the points I make about utterances can be transferred to such "mentalistic" conceptions of logic.

${ }^{4}$ This use of "x substitutes for $y$ " has do be distinguished from one that refers to replacing expressions within the same language.
} 
(SF) $\phi$ substitutes for $\mathrm{u}$ iff $\phi$ is a formula and $\mathrm{u}$ is an utterance and there is at least one $\mathrm{x}$ such that (i) $\mathrm{x}$ is an $\mathrm{X}$ and (ii) $\mathrm{u}$ stands in relation $\mathrm{R}$ to $\mathrm{x}$ and (iii) $\phi$ stands for $\mathrm{x}$.

" $\mathrm{X}$ " and " $\mathrm{R}$ " in (SF) are placeholders for labels referring to certain objects and a corresponding relation, respectively. The best known proposals for specifying " $\mathrm{X}$ " and "R" are "proposition" and "expresses", "sentence-type" and "is a token of", and "utterance" and "is identical with".

If we consider once more the role formulas play in proofs of validity, we can immediately derive an important restriction on what may be proposed as the objects formulas stand for. A central reason for introducing formulas into logical proofs is that proofs of validity are designed to show that an inference is valid not just for any reason, but in virtue of a logical form of its premises and conclusion. By representing logical forms, and only logical forms, of premises and conclusions, formulas guarantee that logical proofs depend on nothing more or less than these logical forms. Formulas therefore are required to stand only for such objects which have a logical form that is represented by this formula. And since formulas must not represent anything else besides logical forms, they will stand for all objects which have a logical form represented by the formula. Using " $X$ " once more as a placeholder for a label which refers to the objects formulas stand for, this amounts to:

(LF) $\phi$ stands for $\mathrm{x}$ iff $\phi$ is a formula and $\mathrm{x}$ is an $\mathrm{X}$ and $\phi$ represents a logical form of $\mathrm{x}$.

Drawing on (LF), the question "What do logical formulas stand for?" may be related to the finding that premises and conclusions are always given as utterances by introducing the following scheme, which is, given (LF), equivalent to (SF):

(S) $\phi$ substitutes for $\mathrm{u}$ iff $\phi$ is a formula and $\mathrm{u}$ is an utterance and there is at least one $\mathrm{x}$ such that (i) $\mathrm{x}$ is an $\mathrm{X}$ and (ii) $\mathrm{u}$ stands in relation $\mathrm{R}$ to $\mathrm{x}$ and (iii) $\phi$ represents a logical form of $\mathrm{x}$.

The question

(Q) What should be filled in for " $\mathrm{X}$ " and " $\mathrm{R}$ " in (S) and (LF)?

can now be used as an explication of "What do logical formulas stand for?" and the less specific "What are the objects of logic?" On the one hand, this takes into account the fact that defending Xs as the objects formulas stand for is of little help if not accompanied by a specification of how utterances which present premises and conclusions are related to these Xs. On the other hand, (S) leaves open the possibility that formulas stand for utterances but, of course, (Q) can also be answered by claiming that formulas stand for something else, say, propositions. In this case, what (LF) and (S) yield can be paraphrased as: A formula stands for all propositions which have a logical form represented by the formula and it substitutes for all utterances which express such a proposition. I take this to be what is asserted by the more colloquial claim that formulas stand for propositions. ${ }^{5}$

\footnotetext{
${ }^{5}$ I leave open the question of whether and how the explication given here can be adapted to theories that reject or substantially reinterpret the traditional account of logical proofs outlined above, as for example LePore and Ludwig (2007).
} 
As made explicit in (S) and (LF), considerations about logical form will play a crucial role in arguments for and against proposed answers to (Q). Several points are worth noting about the relation representing a logical form of as used in this paper. Firstly, logical forms are attributed to Xs in (S) and (LF). Consequently, (S) and (LF) do not entitle us to talk about logical forms of utterances, unless we are going to defend utterances as candidates for X. If a formula substitutes for an utterance, it does not represent a logical form of this utterance, but only a logical form of an $\mathrm{X}$ this utterance is R-related to, for example, a sentence-type this utterance is a token of. In short, formulas represent logical forms of Xs, but they substitute for utterances. Secondly, (S) and (LF) avoid the usual but misleading way of talking about the logical form of an $\mathrm{X}$, which disregards that they generally can be attributed multiple logical forms. Ascribing logical forms is relative to a logical theory, and logical forms can be analysed more or less fine-grained (see Brun 2004, Chap. 13, for more details). If, for example, a premise has a first-order logical form represented by $F a \wedge G a$, it also has a first-order form that can be represented by $\mathrm{Ha}$ and zero-order forms represented by $p$ and by $r \wedge s$. Hence all use of "logical form" is relative to a logical theory. And as a consequence of the central role (LF) plays, this relativity affects the entire discussion of the question about the objects of logic presented here. For the sake of simplicity, I will refrain from indicating this explicitly. Thirdly, using the two-place predicate " $\phi$ represents a logical form of $\mathrm{x}$ " and its converse " $\mathrm{x}$ has a logical form represented by $\phi$ " might suggest that logical forms are entities of some kind, different from both Xs and formulas. This, however, I do not intend to imply. For one thing, I think the notion of logical form is best analysed as referring not to any entities but to certain features of Xs that are relevant to the validity of inferences involving these Xs. This includes, for example, the way Xs can be analysed into constituents belonging to logically relevant categories (e.g. Xs, n-ary predicates and singular terms), and the property of being true or false represented by all formulas (cf. Brun 2004, Chaps. 1.2, 4). Yet the arguments that will be discussed in the following sections do not depend on this position. They are compatible with views that interpret logical forms as entities, patterns instantiated by Xs for example (e.g. Lemmon 1987, pp. 4-5), or think of formulas as not representing but being logical forms (e.g. Sainsbury 2001, p. 35). What points about logical forms are really decisive for answering $(\mathrm{Q})$ can be seen best when we investigate into the reasons why the usual proposals are not viable.

\section{The Usual Proposals}

\subsection{Utterances and Content}

The main reason why utterances may seem a promising answer to $(\mathrm{Q})$ are worries about assigning truth-values to sentence-types. As it has been noted countless times, the truth-value of a sentence-type is often highly context-dependent. "I am mad" may well be true when uttered by the Cheshire Cat, but false when Alice says it. In contrast, utterances are essentially context-bound. Therefore, if they can be attributed a truthvalue, it can be attributed absolutely. (Of course, not all utterances can be attributed a truth value, but only those which are used to perform an appropriate speech act. 
However, utterances that present a premise or a conclusion are presupposed to meet this condition.) This also supports the claim that utterances are the "primary" 6 bearers of truth, while sentences have truth-values only derivatively (e.g. Davidson 1990, p. 309). Now standard logic requires that whatever formulas stand for has exactly one truth-value. In other words, candidates for $\mathrm{X}$ must respect the following condition:

(C1) Xs must have exactly one truth-value.

Having accepted these points, one may be tempted to claim that formulas stand for utterances which have exactly one truth-value. $\mathrm{R}$ could then be specified as the relation of identity. This turns $(\mathrm{S})$ into:

(1) $\phi$ substitutes for $\mathrm{u}$ iff $\phi$ is a formula and $\mathrm{u}$ is an utterance and there is at least one $\mathrm{x}$ such that (i) $\mathrm{x}$ is an utterance and $\mathrm{x}$ has exactly one truth-value and (ii) $\mathrm{u}$ is identical with $\mathrm{x}$ and (iii) $\phi$ represents a logical form of $\mathrm{x}$.

This can be simplified to the equivalent:

(S.U) $\phi$ substitutes for $\mathrm{u}$ iff $\phi$ is a formula and $\mathrm{u}$ is an utterance and $\mathrm{u}$ has exactly one truth-value and $\phi$ represents a logical form of $\mathrm{u}$.

A first difficulty with this line of argument is that it suggests the assumption that if an utterance has a truth-value then it has exactly one such value. But this is not correct if we count all sentence-tokens as utterances. Whether, for example, "I love you" printed on a T-shirt is true still depends on the context, viz. who wears the shirt and who reads the inscription and when. The problem can be eliminated if we take "utterance" to refer to a particular use of a sentence-token (of any physical make-up), what is sometimes labelled a "tokening" (Brandom 1994, pp. 451, 664n10). I will therefore use "utterance" in this sense and presuppose that (S) and its instances are interpreted accordingly.

However, the idea that formulas stand for utterances faces an insurmountable problem. We could not use formulas for proving the validity of even the most simple inferences, such as instances of conjunction elimination. For the validity of an inference formalized by

(2) $p \wedge q \Rightarrow p$

depends not only on its premise being a conjunction, but also on whatever we take the conclusion-formula to stand for occurring in isolation and as a conjunct in the premise. This means that proofs of validity generally depend on formulas standing for something repeatable. ${ }^{7}$ But utterances as such are singular physical objects or

\footnotetext{
${ }^{6}$ See Iacona (2002, Chap. 5.4) for various explications of primacy.

7 The argument can be generalized to all inferences of zero-order logic which do not involve constant truth functions. See Hoyningen-Huene (2004, pp. 209-211).

Nolan (1969) has suggested an interpretation of formulas which would treat (2) as a claim about utterances and their parts: every first conjunct of a true conjunction is true. This interpretation of (2) evades the argument about repeatability by assuming that the conclusion-utterance is a part of the premise-utterance and that generally all inferences formalizable by (2) consist of one (conjunctive) utterance only. However, even if one were ready to adopt such an interpretation of (2), this would be of no help for dealing with inferences that consist of two separate utterances, like all the usual examples of conjunction elimination do.
} 
events, localized in space and time, and can therefore not occur more than once (Grandy 1993, p. 55; Hoyningen-Huene 2004, pp. 116-117). The upshot is that whatever formulas are going to stand for must satisfy this condition:

(C2) It must be possible that two non-identical utterances stand in relation $\mathrm{R}$ to the same $X$.

The argument just given draws on an important point about the relations standing for and representing a logical form of. If a formula contains the same sub-formula more than once, then this repeated sub-formula must stand for the same object. And the same goes for sub-formulas within a sequence of formulas. That means that identity and difference ${ }^{8}$ of whatever formulas stand for count as an aspect of logical form, even though the objects a formula stands for of course do not count as an aspect of logical form (cf. Hoyningen-Huene 2004, pp. 40, 42, 43, 74).

This leads to a restriction on what formulas may stand for. On the one hand, we expect atomic formulas of zero-order logic, such as $p$, to represent only a logical form which is common to all Xs, irrespective of our choice of X. And consequently, we expect such formulas to stand for all Xs and to substitute for all utterances which are R-related to at least one X. On the other hand, this need not be correct for atomic formulas which are sub-formulas of some formula. As an example we may take any formula containing two occurrences of the same sub-formula, say $p \rightarrow p$. We must not allow $p \rightarrow p$ to stand for all Xs having a logical form of a conditional with two constituents which are Xs. Rather, we should interpret $p \rightarrow p$ as standing for all Xs having a logical form of a conditional with two constituents which are identical Xs. In general, if a sub-formula occurs more than once within a formula, then these two sub-formulas must stand for the same X. Analogously, this point also applies to sequences of formulas, such as (2). If single formulas are treated as sequences with one member, the resulting restriction can be framed as follows:

(O) For all sequences of formulas $\Sigma$ and all formulas $\phi$ : if $\phi_{1}$ and $\phi_{2}$ are occurrences of $\phi$ in the members of $\Sigma, \phi_{1}$ stands for an $\mathrm{X} \mathrm{x}_{1}$ and $\phi_{2}$ stands for an $\mathrm{X} \mathrm{x}_{2}$, then $\mathrm{x}_{1}$ and $\mathrm{x}_{2}$ must be identical.

More colloquially, $(\mathrm{O})$ requires that all occurrences of the same formula in a sequence of formulas stand for the same $\mathrm{X}$.

Despite the problems just discussed, there might be some motivation for trying to rescue the claim that formulas stand for utterances. In addition to the reasons mentioned in connection with $(\mathrm{C} 1)$, attributing logical forms to utterances would permit for a simpler explication of the question about the objects of logic, avoiding the complications Xs introduce. Ontological qualms about X-entities may be considered another reason for doing away with them. Finally, it might be possible to relax restriction $(\mathrm{O})$ in such a way that the problems with repeatability can be evaded. In the rest of this section, I will therefore examine an alternative approach to dealing with "What do logical formulas stand for?" based on such considerations.

\footnotetext{
${ }^{8}$ Strictly speaking, there is an asymmetry here. Treating two different Xs as identical may lead to incorrect "proofs" of invalid inferences, whereas treating two occurrences of the same X as different will only result in giving away some proofs of valid inferences.
} 
If logical forms are attributed directly to utterances, $\mathrm{X}$ and $\mathrm{R}$ can be eliminated from $(\mathrm{S})$ and (LF). Taking into account (C1), we get:

(U) $\phi$ substitutes/stands for $\mathrm{u}$ iff $\phi$ is a formula and $\mathrm{u}$ is an utterance and $\mathrm{u}$ has exactly one truth-value and $\phi$ represents a logical form of $u$.

Consequently, distinguishing substituting for from standing for becomes pointless, as (U) shows. Next, we also need to replace $(\mathrm{O})$ with a principle not involving $\mathrm{X}$ :

$\left(\mathrm{O}^{*}\right)$ For all sequences of formulas $\Sigma$ and all formulas $\phi$ : if $\phi_{1}$ and $\phi_{2}$ are occurrences of $\phi$ in the members of $\Sigma, \phi_{1}$ stands for an utterance $\mathrm{u}_{1}$ and $\phi_{2}$ stands for an utterance $\mathrm{u}_{2}$, then $\mathrm{u}_{1}$ and $\mathrm{u}_{2}$ must stand in relation $\mathrm{S}$ (where " $\mathrm{S}$ " is a placeholder for a label referring to an appropriate relation of sameness between utterances).

To avoid the troubles with repeatability, $\mathrm{S}$ must now be specified as a relation of sameness that can hold between utterances that are not identical. Such a relation can be provided by introducing an appropriate notion of "content". One can then say that what matters for the validity of inferences formalized by (2) is not exhausted by the requirement that they all have a premise featuring a conjunction but also turns on the conclusion and the first conjunct having the same content. In short, while content itself does not count as an aspect of logical form, identity and difference of content do. What this exactly amounts to depends on how we define "content".

As a first try, we can experiment with a simple approach and treat contents as a kind of "complement" of logical forms by stipulating that, given a logical form of an utterance, "content" refers to all aspects of this utterance which do not count as aspects of the logical form in question (see e.g. Hoyningen-Huene 2004, p. 11). But once more we could not have any utterances with a logical form represented by, say, $p \vee \neg p$. For the complement of a logical form of an utterance is no more repeatable than the utterance itself. ${ }^{9}$ The only way to evade this problem is to introduce a notion of content which secures the possibility that distinct utterances may have the same content. So the price we have to pay for eliminating Xs is that we now need a substantial account of content.

But there is an additional problem for the claim that formulas stand for utterances. Its range of application is far too restricted. Consider a typical formalization, such as:

(3) Either Alf or Beth will win.

Beth won't win.

Alf will win.

(4) $p \vee q ; \neg q \Rightarrow p$

If we assume that formulas stand for utterances, what exactly do the first $p$ and the second $q$ in (4) stand for? Perhaps one could claim that $p$ stands for the discontinuous

\footnotetext{
${ }^{9}$ Even though the complement-definition of content is of little help if applied to utterances, it may be useful if applied to some other Xs.
} 
utterance "Alf will win", which is partly shared by the utterance "Beth will win". But this seems quite far-fetched and for $q$ there is simply no utterance "Beth will win" to be found in the second line of (3). Suggesting that formulas might also stand for parts of utterances does not really fare better. It seems absurd to claim that the first $p$ stands just for "Alf" and the second $q$ for, well, "Beth w... win"? Another counter to these difficulties is to claim that only entire formulas stand for something, while proper sub-formulas do not. However, this is not only implausible, but such a position would largely give up the original question. In order to avoid that, one could lastly deny that the formulas in (4) stand for the utterances in (3) and claim that strictly speaking they can stand only for utterances such as:

(5) Either Alf will win or Beth will win.

It is not the case that Beth will win.

Alf will win.

This line of argument also calls for a modification of the procedure for proving validity described above. If problems similar to those illustrated by (3) should not exclude most inferences from being formalized, one has to make sure that they are properly set up by preceding argument analysis. This, however, is a rather unwelcome consequence. Delegating a great deal of logical analysis to pretheoretical treatment is too high a price for an answer to the question about the objects of logic, at least as long as there are alternatives.

\subsection{Propositions and Meaning}

The most popular answer to (Q) identifies $\mathrm{Xs}$ as propositions and explains $\mathrm{R}$ as the relation of expression between utterances and propositions:

(S.P) $\phi$ substitutes for $\mathrm{u}$ iff $\phi$ is a formula and $\mathrm{u}$ is an utterance and there is at least one $\mathrm{x}$ such that (i) $\mathrm{x}$ is a proposition and (ii) $\mathrm{u}$ expresses $\mathrm{x}$ and (iii) $\phi$ represents a logical form of $\mathrm{x}$.

However, "proposition" covers many different notions, including some which have originally been introduced under another label, such as "statement" (Strawson 1952) or "thought" (Frege 1918). To simplify matters, I will use "proposition" throughout, ignore terminological differences and arrange the discussion about various notions of propositions according to distinctions which are relevant to their being defensible as answers to (Q). To begin with, we may consider a typical use of the term "proposition", such as:

Now it seems to me as clear as anything can be in philosophy that the two sentences "Socrates is wise", "Wisdom is a characteristic of Socrates" assert the same fact and express the same proposition. They are not, of course, the same sentence, but they have the same meaning, just as two sentences in two different languages can have the same meaning. [...] whichever we say we mean the same thing. Now of one of these sentences "Socrates" is the subject, of the other "wisdom"; and so which of the two is subject, which predicate, depends upon what particular sentence we use to express our proposition, and 
has nothing to do with the logical nature of Socrates or wisdom, but is a matter entirely for grammarians. (Ramsey 1925, p. 12)

Ramsey's idea is clear enough. Roughly speaking, a proposition is the meaning expressed by an utterance. More specifically, the following properties are usually attributed to propositions: (i) They are true or false. (ii) The same proposition can be expressed by different utterances. (iii) Propositions are entities not only different from utterances, but of a different kind. They are not individuated by reference to space and time, and in this sense they are abstract entities. (iv) Utterances express the same proposition if they have the same meaning, say the same thing, or have the same semantic content (in short, if they are "synonymous"). (v) The same proposition can be expressed using sentences which differ linguistically. Properties (i) and (ii) mean that propositions meet conditions (C1) and (C2). Hence they must be adopted by all who defend propositions as the objects formulas stand for.

Important differences between various notions of propositions relate to properties (iii), (iv) and (v). What kind of entities are propositions? And in particular, are they structured? Under what conditions do utterances count as expressing the same meaning or saying the same thing? What does indifference to linguistic variance amount to? In the present context, the last two questions are the crucial ones. They concern the relation $\mathrm{R}$ between utterances and the propositions they express; that is, the conditions which decide whether two utterances count as the same $X$ if propositions are going to play the role of $\mathrm{X}$. In principle, this issue is independent of the ontological question about the nature of propositions. Specifically, the conditions that determine whether two utterances express the same proposition can be sensitive to the linguistic structure of the utterances, even if propositions themselves have a completely different structure or no structure at all. In this section, I shall concentrate on the idea that equivalence in meaning is a sufficient condition for identity of proposition expressed and that this implies that utterances which are not utterances of the same sentence can express the same proposition in virtue of being logically equivalent or of featuring synonymous expressions (see Sect. 2.4 for other notions of proposition). ${ }^{10}$ In Carnap's (1956, Sects. 6, 14) terminology, two utterances express the same proposition if they are L-equivalent (in contradistinction to: if they are intensionally isomorphic). The probably bestknown account of propositions with these properties is Stalnaker's $(1976,1984)$ proposal to identify propositions with functions from possible worlds to truth-values or with sets of possible worlds. ${ }^{11}$ Alternative accounts rely on other criteria for the identity of propositions, such as: two utterances express the same proposition if they have identical truth-conditions, assertibility-conditions or inferential role.

Understood this way, propositions promise to do away with all aspects of utterances which are logically irrelevant, specifically, properties of physical realization as well as peculiarities of linguistic expression. Instead they partition

\footnotetext{
${ }^{10}$ Frege explicitly formulated this criterion, among others (see Penco 2003).

11 Whether Stalnaker himself would propose to answer (Q) with reference to propositions is less clear. On the one hand, he takes propositions to have logical structures and to stand in logical relations, such as entailment (1984, p. 10). On the other hand, he treats statements about logical equivalence as a statements about relations between sentences (1984, p. 72).
} 
utterances into semantically relevant equivalence classes. The trouble with the identity criteria mentioned is that they have a tendency to be too generous. Two problems shall be mentioned (cf. Grandy 1993, pp. 53-54).

Firstly, according to the criteria mentioned both of the couples

(6.1) Jack went up the hill and Jill went up the hill.

(6.2) Jill went up the hill and Jack went up the hill.

(7.1) Not all Martians are green.

(7.2) Some Martians are not green.

consist of two utterances expressing the same proposition. But there is an important difference between these and Ramsey's example. The equivalence in both (6) and (7) clearly has not only "grammatical" but logical reasons, commutativity of conjunction in (6) and relations between quantifiers and negation in (7). This raises the following problem: if formulas stood for propositions, then all equivalences would have a trivial proof; that is, we would never need more than a proof that all instances of

$$
\phi \Leftrightarrow \phi
$$

are valid. This undesirable result is not restricted to proofs of equivalence, but can similarly affect other proofs as well. One could, for example, bypass first order logic in the proof of

(9) If not all Martians are green, there are minorities on Mars.

Some Martians are not green.

There are minorities on Mars.

by treating it as an instance of modus ponens. If we are not ready to accept such unnecessarily trivial proofs, we must not treat logical equivalence as a sufficient condition for identity of logical form (cf. Davidson 1980, p. 145).

A second problem arises if we assume, for example, that in virtue of synonymy the following two utterances express the same proposition:

(10.1) Donald is a drake.

(10.2) Donald is male duck.

Then we could formalize

(11) Donald is a drake.

(using $F, G$ and $a$ for "male", "duck" and "Donald" respectively) as

(12) $F a \wedge G a \Rightarrow G a$

and "show" the validity of (11) by using conjunction elimination alone. Unless one is willing to accept the claim that all valid inferences are formally valid (as proposed in, for example, Wittgenstein 1922), this constitutes a fatal problem for the position that formulas stand for propositions. It opens the door to mock proofs for materially valid inferences. While in the preceding examples logical proofs are trivialized, we 
here face the problem that propositions enable us to show inferences as truth functionally valid which we consider to be truth functionally invalid.

The conclusion to be drawn is that we cannot understand formulas as standing for propositions in the sense discussed here. The criteria deciding whether two utterances express the same proposition would force us to accept unnecessary trivial and even incorrect proofs. The reason for this failure is that some of the allegedly "grammatical" features of utterances are in fact relevant to logic, but the notions of proposition under consideration ignore them. As a further condition for answering (Q) we can therefore introduce:

(C3) Logical equivalence and synonymy of two non-identical utterances must not be sufficient conditions for their standing in relation $\mathrm{R}$ to the same $\mathrm{X}$.

It is important to note that the appeal to logical equivalence in (C3) introduces an implicit reference to the logical theories we consider relevant to the application of (C3). For example, the above argument against treating (9) as an instance of modus ponens turns on the fact that a more sophisticated analysis is available in first order logic. But if we imagine that propositional logic was the only logical theory we had, proving (9) as an instance of modus ponens would be as acceptable as using the same formula for the following two utterances (cf. Davidson 1963, p. 349):

(13.1) Jack and Jill went up the hill.

(13.2) Jack went up the hill and Jill went up the hill.

Additionally, the formulation of (C3) is relatively cautious. It leaves open the possibility that under certain conditions two equivalent or synonymous expressions can count as the same $\mathrm{X}$. The reason is that in standard practice of first-order logic not all synonymous expressions are treated in the same way. Whereas proving (11) by means of (12) is considered unacceptable, it is routine to follow Frege (1918, pp. 63-64) in formalizing inferences like (14) with (15):

(14) Every horse is an animal.

Rosinante is a steed.

Rosinante is an animal.

$\forall x(F x \rightarrow G x) ; F a \Rightarrow G a$

One may well wonder whether this practice can be justified by a sound principle. However, in the present context that issue may remain unresolved, since no stronger principle than (C3) is needed for ruling out answers to (Q) which lead to the "proofs" we found objectionable.

\subsection{Sentences and Linguistic Structure}

The arguments presented in the previous section have shown that at least some linguistic differences between equivalent utterances are relevant to the question whether they should count as the same $\mathrm{X}$. This provides some motivation for proposing that formulas stand for sentence-types (sentences, for short). Sentence- 
types seem to be promising, since qua types they are repeatable and qua sentences they are linguistic entities which do not eliminate the differences in structure we found being relevant to logic. But of course not all sentences qualify as objects formulas may stand for, but only those which are true or false $(\mathrm{C} 1)$. The claim that formulas stand for sentences is therefore to be understood as restricted to sentences which have exactly one truth-value according to some criterion (T):

(S.S) $\phi$ substitutes for $\mathrm{u}$ iff $\phi$ is a formula and $\mathrm{u}$ is an utterance and there is at least one $\mathrm{x}$ such that (i) $\mathrm{x}$ is a sentence-type and $\mathrm{x}$ has exactly one truth-value according to (T) and (ii) $\mathrm{u}$ is a token of $\mathrm{x}$ and (iii) $\phi$ represents a logical form of $\mathrm{x}$.

Before going into any detail, it may be worth noting that explicating the typetoken relationship is far from trivial (see e.g. Goodman 1976, Chaps. IV.2-3; Kaplan 1990, pp. 96-110). Nevertheless, I will follow common practice and presuppose that an utterly unambitious syntactical criterion like the same words in the same order does the job of deciding whether two utterances are tokens of the same type. After all, the problem of an exact definition of "sentence-type" is a rather pervasive one. It affects most theories which refer in some way to utterances and in particular all proposals for answering (Q), as soon as one tries to specify the relation $\mathrm{R}$ between utterances and the objects formulas stand for (unless " $\mathrm{X}$ " and "R" are specified as "utterance" and "is identical with").

Proposing sentences as Xs faces two classes of problems, one well-known, one mostly overlooked. The first class of problems concerns the specification of $(\mathrm{T})$. It is the basis for a popular objection against sentences which draws on (C1). The argument is mostly the same as the one which has already been portrayed as supporting utterances. Since the truth-value of a majority of sentences is contextdependent, their truth-values — if they are going to have a truth-value at all-must be derived from utterances by appeal to a principle such as:

(T.1) A sentence is true iff all its utterances are true and it is false iff if all its utterances are false. $^{12}$

But this means that most (or even all) sentences cannot be assigned a truth-value, because either some of their utterances cannot be classified as true or false, or not all their utterances have the same truth-value.

The first difficulty is mainly caused by vague or ambivalent utterances which cannot straightforwardly be assigned a truth-value. The examples are notorious:

(16) The journey from Rome to Vienna takes a long time. (How much time is "long"?)

(17) I've forgotten about the bill. (An invoice, a theatre program, an act of legislation?)

\footnotetext{
${ }^{12}$ Some defenders of propositions claim that utterances have truth-values only in virtue of expressing propositions. This turns (T.1) into: A sentence is true iff all its utterances express a true proposition and it is false iff if all its utterances express a false proposition. Since the following arguments do not depend on whether utterances have their truth-value ascribed directly or derived from the propositions they express, I will confine myself to the simpler picture without propositions.
} 
The second difficulty arises because for most sentences the truth-value of their tokens depends on (linguistic and factual) context. Therefore, there is in general no guarantee that they have the same truth-value. This is most obviously the case if an utterance contains indexicals, such as "here", "yesterday", "you", "this" etc, but it also concerns, for example, most sentences containing proper names. ${ }^{13}$ Whether

\section{(18) George is president.}

is true, depends, inter alia, on who "George" refers to in this particular utterance.

Quine has defended two solutions to these problems with (T.1). The more radical one consists in resorting to "eternal sentences" (Quine 1960, Sect. 40; foreshadowed in Frege 1969, pp. 146-147/134-135). This amounts to claiming that formulas cannot stand for all sentences, but only for those which qualify as true or false according to (T.1); that is, for sentences which are neither ambiguous nor vague nor context-dependent. Consequently, in most cases utterances have to be prepared before logical analysis can start. Indexicals have to be tuned up with contextual information and ambiguous or vague expressions have to be eliminated. This idea faces at least three problems. It is far from clear that indexical elements, vagueness and ambiguity can always be completely eliminated. More importantly, it is not clear under what conditions exactly a sentence counts as free from context dependency, vagueness and ambiguity. Finally, it has been argued (e.g. Perry 1979) that there are "essential indexicals", which cannot be eliminated without a substantial change in meaning.

Fortunately, we do not need to enter into these questions, since the strategy of eternalizing is overkill anyway. As Quine (1960, p. 227, 1982, pp. 55-57) has argued, the problems challenging sentences can be solved without insisting on them being unaffected by vagueness, ambiguity and context dependency. Take for example the two inferences:

(19) If this is true, then I am a nurse.

This is true.

I am a nurse.

\section{Nice food is not cheap. \\ Cheap food is not nice.}

It is irrelevant to the formal validity of (19) what the referents of "I" and "this" are, as long as they are guaranteed to remain constant within (19). Similarly, we do not need to know what counts as "nice" or as "cheap" food in order to establish the validity of (20), as long as we can assume that the terms are used in the same way in premise and conclusion. The reason is, that logical validity of an inference is solely dependent on its logical form. This means that (19) can be understood as an instance of modus ponens, provided the "content" of the two occurrences of "this is true" and "I am a nurse" is guaranteed to be the same, irrespective of what this

\footnotetext{
13 The category of indexicals is here to be understood in a broad sense, so that it includes not only expressions whose meaning is dependent on the circumstances of utterance, but also relative pronouns and other anaphoric or cataphoric expressions.
} 
content is. ${ }^{14}$ Analogously this is true for the terms and their referents in (20). The upshot is that we do not need to eliminate context-dependency, vagueness and ambiguity; it is enough to eliminate equivocation within the context of an inference. This makes it possible for formulas to stand for all sentences, as long as we can guarantee that no equivocation results. The proposal can be worked out in two steps. First, we generally ban the fallacy of equivocation:

(E) Within an inference, tokens of the same type must have the same semantic value.

Secondly, we replace (T.1) by

(T.2) In the context of an inference $i$, a sentence is true iff all its utterances in i are true and it is false iff all its utterances in $\mathrm{i}$ are false.

Taken together, (E) and (T.2) promise to turn sentences into a candidate for answering (Q) which meets all conditions discussed so far. It may in certain cases be difficult to enforce $(\mathrm{E})$, but it is, by orders of magnitude, more realistic than insisting on eternal sentences. However, this solution comes at a price. Since (T.2) is relativized to the context of an inference, working out this proposal requires to relativize "substitutes for" in (S) and (S.S) as well. And whether a given sentence is something a formula can stand for can be decided only within the context of an inference. ${ }^{15}$ This price may well be worth paying but, unfortunately, $(\mathrm{E})$ is rather superficial. It is too strong, for we need not require that all tokens of the same type have the same semantic value, but only corresponding ones. Applying (E) to "it" in the following would be nonsense:

(21) If it is getting cold I will either cover the exotic plants myself or convince you to do it.

However, (E) is also not strong enough, for it addresses neither syntactical ambiguities nor ambiguities of expressions directly relevant to logical form. (Many textbooks treat "either ... or ..." in sentences such as (21) as inducing an ambiguity of logical form, but see LePore 2000, pp. 77-79.) As a consequence, (E) needs to be replaced by a more ambitious principle which refers (at least, see Grandy 1993, pp. 57-58) to syntactic structures of sentences.

But once more, we do not need to address these problems here, because there is a second, independent class of problems challenging sentences as the objects formulas stand for. In spite of being much simpler, these problems have got much less attention (cf. again Grandy 1993, pp. 58-60). All proposals considered so far use a notion of sentence that has identity criteria which are far too fine-grained for the purpose at hand. For example:

\footnotetext{
14 Just some guarantee that both occurrences have the same truth-value is not enough. Even if all utterances of, for example, "I am here now" must be true regardless of context (Kaplan 1989), it does not follow that all inferences from one utterance of this sentence to another one can be formalized as instances of $\phi \Rightarrow \phi$. In fact, such a formalization constitutes a fallacy of equivocation unless the two utterances are produced simultaneously by the same person.

15 We could therefore say, that being an $\mathrm{X}$ is a role sentences can play, quite analogous to other roles they may play, such as being a premise or a conclusion.
} 
(22) If the letter is not stamped you will be charged extra.

The letter is unstamped.

You will be charged extra.

If we did take formulas as standing for sentences, then (22) could not count as an instance of modus ponens. For the two inscriptions in italics are not tokens of the same sentence-type according to any orthodox definition of sentence-type. Matters get even worse if we turn to a language with a systematic difference in word order between main clauses and subordinate clauses. It is, for example, extremely difficult to find even one example of an inference instantiating modus ponens in German (cf. Frege 1969, pp. 281/261-262), if we assume that formulas stand for sentences:

(23) Wenn Schnee weiß ist, dann ist Gras grün.

Schnee ist weiß.

Gras ist grün.

It is quite obvious that these two examples are only the tip of an iceberg (inference (3) is another case in point). The position that formulas stand for sentences simply is not compatible with standard logical practice. The reason is that logically completely irrelevant aspects of linguistic expression count as differences in sentence-type. This result is just the opposite of what propositions do, namely, eliminate all "grammatical" differences. So we still face the challenge to find criteria for treating utterances as R-related to the same $\mathrm{X}$ which allow us to ignore some, but not all linguistic differences.

One intuitively appealing strategy for coping with the problems just discussed is to resort to syntax for introducing some entities which are closely related to sentences but whose criteria of identity are not as sensitive to linguistic detail as those of sentence-types. It is, for example, reasonable to expect that a better result could be achieved by uniting sentence-types which can be derived from a common syntactic representation or from one another by certain syntactical transformations. Although such an approach could eliminate the problems exemplified in (23), syntax alone will not suffice for dealing with problems like (22). Semantical information about the meaning of specific words is involved in deciding that (22) is an instance of modus ponens, in contrast to, for example:

(24) If the dock workers are unionized they will join the strike.

The dock workers are not ionized.

The dock workers will join the strike.

All in all, one may hope that with a more or less full-fledged linguistic analysis one would be in a position to decide whether two sentences count as the same X.

Nevertheless, linguistic analysis as such is not what we are after when trying to answer (Q). What we really need to know at this point is: how can we distinguish logically relevant from logically irrelevant features of linguistic expression? We therefore need, in addition to a sufficiently detailed linguistic analysis of sentences, some rules for deciding on basis of such an analysis whether two sentences count as the same $X$. And which linguistic features are relevant to such decisions depends on 
the logical theories we are taking into account. So what is really needed is a proposal that respects the following condition:

(C4) Logically irrelevant linguistic differences between utterances must not be a sufficient condition for their not standing in relation $\mathrm{R}$ to the same $\mathrm{X}$.

As (C3), condition (C4) is to be understood as implicitly relative to the logical theories we consider relevant to its application.

The problem with condition (C4), of course, is that it is useless without spelling out what "logically relevant" means. This challenge can be made more clear, if we make use of the fact that one rationale for introducing (LF) and (S) was to secure that a formula stands for exactly those Xs which have a logical form represented by that formula. Since logical forms are all that matters for the relationship between formulas and Xs, we can identify the logically relevant linguistic differences between sentences as those differences which, in a given logical theory, count as aspects of logical form. Equivalently, we can define that two sentences must count as different Xs if and only if their tokens cannot all be given identical adequate formalizations. It thereby becomes clear that working out the details behind $(\mathrm{C} 4)$ amounts to giving a theory of formalization. I take this to be a strong reason against the strategy of expecting that linguistics is bound to come up with a viable proposal for answering (Q) - unless it is at least implicitly guided by logical considerations; that is, it is a theory of formalization in linguistic terms.

This interconnection between $(\mathrm{Q})$ and formalization becomes even more apparent if we investigate some further strategies for answering (Q) drawing directly upon logic.

\subsection{Propositions and Logical Form}

Several proposals are available if we seek an answer to (Q) based on logical considerations. I will discuss three closely related approaches. A first option is to focus on logical practice and ask, for example: if we are given a formula, how do we go about finding out what utterances it substitutes for? For this process, which I call "verbalization", one can follow a fairly established recipe. Kalish et al. (1980, pp. 9-11, 53-57, 129-130, 209-211, 264-266, 315-316) give a description which involves three steps. If, for example, we have

$$
\forall x(F x \rightarrow G x) \rightarrow \neg p
$$

we first need to decide how the non-logical symbols should be interpreted. So we need a correspondence scheme; that is, a table that specifies for each non-logical symbol a unique reading. ${ }^{16}$ For example:

(25.1) Fx: $x$ is a whale; $G x: x$ is a mammal; $p$ : Moby Dick is a fish

For reasons that soon will become obvious, I call an ordered pair of a formula and a correspondence scheme a "formalization".

\footnotetext{
16 "Correspondence scheme" is Sainsbury's term (2001, p. 51). Kalish et al. use "scheme of abbreviation" (1980, p. 8).
} 
In the second step, the formalization is "read". The result (understood as a type rather than a token) is called a "literal verbalization":

(25.2) (If for each $\mathrm{x}$ (if $\mathrm{x}$ is a whale, then $\mathrm{x}$ is a mammal), then it is not the case that Moby Dick is a fish).

Most literal verbalizations cannot count as sentences of an ordinary language because they are grammatically deviant or include remains of logical symbolism, such as brackets for grouping and variables for cross-referencing. But we can, in a third step, paraphrase a literal verbalization to get an ordinary language expression, which is a "free verbalization":

(25.3) If all whales are mammals, then Moby Dick is not a fish.

One should, of course, go on and define more exactly the two notions of verbalization involved. This is relatively easy for literal verbalizations. In fact, Kalish et al. give effective rules for literal verbalizations. And in principle, there is no reason why this could not be done in a way that secures that every formalization has exactly one literal verbalization. However, to give a definition of "free verbalization" seems pretty hopeless. It would amount to giving a precise account of the notoriously vague term "paraphrase".

Nevertheless, it is instructive to see how these notions could be used for answering (Q). The obvious idea is to claim that a formula substitutes for all utterances which are tokens of a literal or free verbalization of some formalization with this formula. Since all verbalizations are paraphrases of literal verbalizations, this amounts to:

(S.V) $\phi$ substitutes for $\mathrm{u}$ iff $\phi$ is a formula and $\mathrm{u}$ is an utterance and there is at least one $\mathrm{x}$ such that (i) $\mathrm{x}$ is a literal verbalization of some formalization with formula $\phi$ and (ii) $\mathrm{u}$ is a token of a paraphrase of $\mathrm{x}$.

To meet requirement $(\mathrm{O})$ we may insist that sequences of formulas are verbalized using one correspondence scheme only. (S), on the other hand, yields (26) if " $\mathrm{X}$ " is specified as "is a literal verbalization of some formalization" and " $\mathrm{R}$ " as "is a token of a paraphrase of":

(26) $\phi$ substitutes for $\mathrm{u}$ iff $\phi$ is a formula and $\mathrm{u}$ is an utterance and there is at least one $x$ such that (i) $x$ is a literal verbalization of some formalization and (ii) $u$ is a token of a paraphrase of $\mathrm{x}$ and (iii) $\phi$ represents a logical form of $\mathrm{x}$.

This, however, is equivalent to $(\mathrm{S} . \mathrm{V})$, provided we accept that formulas represent logical forms of "their" literal verbalizations:

(27) $\phi$ represents a logical form of $x$ iff there is a $y$ such that (i) $y$ is a formalization with formula $\phi$ and (ii) $\mathrm{x}$ is a literal verbalization of $\mathrm{y}$; in short, iff $\mathrm{x}$ is a literal verbalization of some formalization with formula $\phi$.

As candidates for answering (Q), literal verbalizations are an improvement on sentences. They are on a par with respect to repeatability (C2), and assigning truthvalues to literal verbalizations leads to the same questions as it does with sentences (C1). If we leave aside the issue of (E) and (T.2), two important points remain. 
Firstly, literal verbalizations can be different but yet logically equivalent (C3) if they are introduced by rules that guarantee that different literal verbalizations are assigned to structurally different formulas (cf. the rules in Kalish et al. 1980). Secondly, nothing stands in the way of defining the relation $\mathrm{R}$ between utterances and literal verbalizations in such a manner that logically irrelevant linguistic differences between utterances do not imply that they have to be assigned to different literal verbalizations (C4).

Now this proposal will be as good as the explication we can give for "paraphrase" (as well as for "correspondence scheme" and "literal verbalization"). Furthermore, we do not need just any account of paraphrasing, but one which gives us the right results when used in (S.V). This is certainly not the case if we take the term "paraphrase" as ordinarily used. For in this sense, equivalences, such as

(7.1) Not all Martians are green.

(7.2) Some Martians are not green.

are perfect examples of paraphrases. Besides not being properly defined, such a notion of paraphrase would threaten to lead us into exactly the same problems as we encountered with propositions. Instead, we need a notion of paraphrase which is sensitive to logical form but does not rely on logical equivalence or synonymy. All in all, it seems that there is really not much to gain from turning to verbalizations. For sure, literal verbalizations allow for precise definition, but introducing paraphrases results in replacing the question "What do logical formulas stand for?" with "What counts as a paraphrase of a literal verbalization?" Since literal verbalizations are little more than verbose formulas, there is really not much of a difference between this question and "What counts as an adequate formalization of an utterance?"

This point can be reinforced if we turn to the second approach, switch our perspective and ask how in the common practice of logic one finds out which formulas can be substituted for a given inference; in short, how one "formalizes". There is a striking analogy between the process of verbalization and the procedures of formalizing which are described in textbooks by those authors who care about formalization (e.g. Epstein 2001; Lampert 2005; see also Blau 1977). Indeed, what they suggest comes very close to inverting verbalization. According to such explanations, formalization can be done, and often is done, in two steps. Firstly, the utterance in question is replaced by an expression which is more perspicuous with respect to logical form. Secondly, a formula is assigned to the "explicit version" or "standardized text" resulting from the first step. There are various proposals on offer for the first step. While, for example, Blau (1977, pp. 4-6) gives a relatively informal characterization, Lampert (2005, Chaps. 3.1, 9.1) and Kalish et al. (1980; see beginning of this section) provide at least rules that determine which expressions count as explicit versions, although these rules are silent on the question which expressions count as explicit versions of which utterances. Additionally, Lampert gives rules for the second step from explicit versions to formulas.

For later use, we may note additionally that the result of formalizing should be taken to be a formalization, not only a formula, or else it would not generally be possible to tell adequate formalizations from inadequate ones. The following, for 
example, becomes inadequate if $p$ and $q$ are switched either in the correspondence scheme or in the formula (but not in both), as well as if one of the sentences in the correspondence scheme is replaced by another one, such as "Vladimir swears":

(28) Pozzo loves smoking, but Estragon does not.

(29) $p \wedge \neg q \quad p$ : Pozzo loves smoking; $q$ : Estragon loves smoking

Without going into further details of the above-mentioned accounts of formalization, it quickly becomes clear that explicit versions will turn out not to be substantially different from literal verbalizations as soon as one tries to define them strictly (this is explicitly so in Kalish et al. 1980, see pp. 11-12). Moreover, the first step obviously consists in paraphrasing once more. Consequently, these procedures of formalization really boil down to the converse of verbalization and we do not get a new answer to (Q). The crucial problem with explicit versions is the same as with literal verbalizations: no detailed account of the relationship $\mathrm{R}$ between utterances and explicit versions (or literal verbalizations) is available.

Similar virtues and vices can be found in many theories of propositions, which are the third approach discussed in this section. The currently prevailing view is that propositions are entities which are not only true or false, but structured in a logically relevant way (see e.g. the survey in King 2005). In the most simple cases, their structures are just logical forms, as for example in Carnap's (1956, Sect. 14) account based on intensional isomorphism. More often, these structures are not logical forms of standard first-order logic, but more fine-grained relatives thereof (e.g. Cresswell 1985; Soames 1987; Zalta 1988; Menzel 1993). Other theories exploit Chomsky's LF (Logical Form), a syntactic structure closely related to logical form (e.g. Higginbotham 1991; Larson and Ludlow 1993; King 1995). ${ }^{17}$ The essential point for present purposes is that all these theories use criteria for assigning propositions to utterances which are sensitive to logical forms. Consequently, there are important differences to the accounts discussed in Sect. 2.2, although the resulting proposal to answer (Q) is again framed in terms of propositions expressed by utterances, just as in (S.P). The concepts of proposition mentioned here, meet not only (C1) and (C2), but (C3) as well. In fact, differentiating between logically equivalent utterances is a major motivation for introducing these notions in the first place. Nevertheless, as potential answers to (Q) they are facing two challenges. ${ }^{18}$

First, recent theories of propositions introduce this notion not primarily as an answer to (Q), if at all, but as the objects we relate to in propositional attitudes. But this use of propositions is irrelevant for the question whether they can be defended

\footnotetext{
17 Importantly, even though LF is closely related to logical forms of generalized quantifier theory, it is a syntactic structure which is part of Chomsky's program of naturalizing linguistics. As such it answers to principles which are not logical: properties of LF are "not to be settled in terms of considerations of valid inference and the like" (Chomsky 1986, p. 205n, cf. pp. 67, 156). It therefore is an empirical question, whether and in what respects Logical Forms can be equated with logical forms, in spite of widespread use of LF for addressing logical problems.

18 There are also theories which hold that propositions themselves are unstructured entities even though logical forms play a crucial role in deciding what proposition(s) an utterance expresses (e.g. Bealer 1993). If this implies that propositions do not have logical forms, they cannot be what formulas stand for according to (LF). If not, such unstructured propositions raise the same problems as those structured by logical forms.
} 
as an answer to (Q). Moreover, it leads to problems with condition (C4) (cf. Richard 1990, pp. 171-173; Salmon 1992; King 1996). The reason is that identity conditions for propositions as objects of propositional attitudes are extremely fine-grained and include aspects of utterances which are standardly considered irrelevant to logical inference when these utterances are not used in propositional attitude-contexts. (30) and (31), for example, each consist of a pair of utterances which accepted practice counts as the same $\mathrm{X}$ while denying that they entail each other when prefixed by "Alfreda believes that":

(30.1) Rosinante is a horse.

(30.1) Rosinante is a steed.

(31.1) The letter is not stamped.

(31.1) The letter is unstamped.

Consequently, for answering (Q) we need an account of propositions that is not tailored to propositional attitude-contexts and the fine-grained distinctions they require, but one that takes, for example, standard first-order logical form as a criterion for deciding whether two utterances express the same proposition. In fact, I think, this is what logicians usually have in mind when they claim that formulas stand for propositions. And this proposal comes extremely close to the first two approaches discussed earlier in this section. As long as we restrict ourselves to question (Q), I can see no reason why one should not treat literal verbalizations as standard expressions of propositions or simply identify propositions with literal verbalizations.

Irrespective of such an identification, most theories of propositions leave us with a second problem, quite similar to the difficulties with literal verbalizations and explicit versions. As the two placeholders " $\mathrm{X}$ " and " $\mathrm{R}$ " in (S) make explicit, answering (Q) involves a double task. It requires us to identify not only some objects $\mathrm{X}$, but also to specify their relation $\mathrm{R}$ to utterances. Therefore, proposing some notion of proposition as an answer to (Q) is not enough. As long as the relation $\mathrm{R}$, expresses, is not spelled out in sufficient detail, such a proposal does not much more than giving the problem a new label. This lacuna affects most current theories of propositions, the major exception being accounts derived from Chomsky's theory of LF, such as Larson and Segal (1995).

A great deal of mainstream logic takes this as a motivation to stick to answering (Q) with propositions structured by logical forms and to simply admit that we do not have a sufficiently detailed account of a corresponding relation R. After all, many, or perhaps most, logicians are simply convinced that it is extremely difficult or even impossible on principled grounds to come up with anything like a precise account of formalization; that is, of how logical formulas can be assigned to expressions of ordinary language. Given that propositions structured by logical forms are close relatives of formulas in the same manner that literal verbalizations and explicit versions are, the difficulties in explaining the relation of any of these to utterances are comparable. There is an obvious reason for this being so: insofar as propositions have a structure which cannot be defined without, at least implicitly, appealing to logical forms, they are not only closely related to formulas, but simply parasitic upon formalizations; without the help of a logical formalism we had no clue about 
how to define them. In fact, the two projects of answering (Q) with appeal to logically structured propositions and of giving an account of formalization are so closely related, that the solution of one problem could be used for solving the other one. If we had an account not only of the relation $\mathrm{R}^{*}$ between propositions and formalizations (analogous to the rules in Kalish et al. 1980; Lampert 2005), but of R as well, then we could straightforwardly introduce the following definition: A formalization $\Phi$ is an adequate formalization of an utterance $u$ if and only if there is a proposition $\mathrm{p}$ such that $\mathrm{u}$ stands in relation $\mathrm{R}$ to $\mathrm{p}$ and $\mathrm{p}$ stands in relation $\mathrm{R}^{*}$ to $\Phi$ (cf. Kalish et al. 1980, pp. 11-12). On the other hand, if we had a theory of formalization, we could either use it to complement a theory of propositions (more on this in Sect. 3.3) or we could draw on the idea of tying propositions to literal verbalizations and define: An utterance $u$ expresses a proposition $p$ if and only if $p$ is a literal verbalization of an adequate formalization of $u$. Since utterances generally have more than one adequate formalization, this would imply that they also express several propositions. Consequently, different formula substituting for the same univocal utterance could stand for different propositions. This is not a problem for proving validity, even though for independent reasons one may seek an account which relates univocal utterances to one proposition only. ${ }^{19}$

Strategically, this leaves us with two options for (not) dealing with question (Q). Standard practice of logic suggests that we settle for a modest program of logic which takes logical analysis of ordinary language inferences as largely based on intuitive skills of interpretation and paraphrase. We may still claim that formulas stand for propositions, but we have to admit that this does not amount to an informative answer to (Q), since we have given up on trying to explain how ordinary language utterances are related to propositions and to formulas. Clearly, this widespread stance also abandons the traditional program of logic which aspires to develop a theory that can show the validity of inferences in ordinary language by means of logical proofs. Implicitly, this program is committed to providing a theory of formalization, since without that we have no theoretical support for claiming that a proof carried out in a logical formalism gives us reason to accept a conclusion of an inference in ordinary language. Alternatively, we may accept the challenge of addressing the relationship between ordinary language and logical formulas. In this case, the core problem is to spell out conditions for a formula to represent a logical form of an $\mathrm{X}$ an utterance is R-related to. In short, a satisfactory answer to (Q) presupposes a theory of formalization. Since a reasonably extensive treatment of theories of formalization lies outside the scope of this paper (but see Brun 2004), the following section will be restricted to a discussion of what can be learned from the preceding arguments for a theory of formalization and what consequences for answering (Q) could be drawn from such a theory.

\section{Formalization}

In this paper, I use the term "theory of formalization" in a broad sense for accounts which put us in a position to decide whether some sequence of formalizations counts

\footnotetext{
19 In Brun 2004, Chap. 13, I argue that in a certain sense all adequate formalizations of an utterance in a given logical theory can be interpreted as representing aspects of a single logical form.
} 
as an adequate formalization of some given sequence of utterances (typically an inference). I take this to cover theories that provide criteria of adequacy, as well as procedures which can be used to find adequate formalizations for given utterances. ${ }^{20}$

\subsection{Referring to Utterances}

So what can we learn for a theory of formalization from the merits and pitfalls of the proposals for (Q)? First of all, such a theory must not repeat the fatal mistakes found in some of the proposals discussed. Here is one way how this could happen. On the one hand, a theory of formalization cannot deal with particular utterances one at a time, for the simple reason that doing so would not count as giving a theory. On the other hand, most of what we can attribute to an utterance clearly is not relevant to its formalization (colour of ink, whether you have approved of it and so on). Giving a theory of formalization therefore calls for an answer to the question:

(F) What kind of description should a theory of formalization use to refer to utterances?

Answering (F) amounts to deciding what information about an utterance is needed for determining which formalization(s) should count as adequate. As a consequence, any two utterances which differ only in information not included in the kind of description chosen will be indistinguishable for the theory of formalization and therefore cannot have different adequate formalizations. Question (F) may look conspicuously related to (Q), since possible answers include claims like: a theory of formalization should refer to utterances as tokens of sentences, because all that matters for formalizing an utterance is what sentence it is a token of. But nevertheless, (F) and (Q) are distinct questions. This becomes clear when we go through some possible answers, beginning with those already considered for (Q).

Firstly, a theory of formalization must refer to utterances in a way that does not beg substantial questions about their adequate formalizations. Otherwise the theory will just be more or less trivial. That is, any answer to $(\mathrm{F})$ must be given without explicit or implicit reference to logical forms of Xs utterances are R-related to. This immediately rules out the notions of proposition (and literal verbalizations and so on) discussed in Sect. 2.4 as answers to $(\mathrm{F})$.

Propositions understood as meanings fare no better. The reasons for rejecting them are the same as in Sect. 2.2. If we forced a theory of formalization to accept the same formalizations as adequate for all utterances expressing the same proposition, we again would have to face trivial proofs for equivalences and mock proofs for formally invalid inferences.

For sentences, there were two types of problems. Firstly, the type-token-relation was found to be sensitive to linguistic differences irrelevant to logical form. In other words, we have to allow that a formula substitutes for tokens of different sentences.

\footnotetext{
${ }^{20}$ Classical starting points for procedures and theories of formalization are Montague (1970) (for an application to extensional first-order logic see Link 1979, pp. 242-245) and Davidson (1984, 1980). A third notable paradigm proceeds from Chomsky's $(1986,1995)$ syntactical theories of LF, but see note 17. An important discussion of criteria can be found in Blau (1977, Chap. I.1). Most of what follows can also be applied to informal guidelines for formalizing as presented in e.g. Epstein (2001) and Sainsbury (2001).
} 
But this poses no problem for a theory of formalization. The arguments considered in Sect. 2.3 imply only that if a theory of formalization deals with utterances qua sentences, then it must admit that the same formalization may be adequate for utterances of different sentences. Secondly, there were also problems related to the requirement $(\mathrm{C} 1)$ that whatever formulas stand for must have exactly one truthvalue. This also is a difficulty for a theory of formalization. For if we refer to utterances qua tokens of sentences, we are not in a position to say that a formalization may be adequate for some but not all utterances which are tokens of the same sentence. As the arguments in Sect. 2.3 have shown, the core of this problem is equivocation, which thereby also becomes crucial for sentences as answers to $(\mathrm{F})$.

\subsection{Dealing with Equivocation}

Choosing a strategy for addressing equivocation calls for several methodological decisions. A first approach is based on the conviction that dealing with equivocation is not a genuinely logical task and therefore proposes to hand it over to some preliminary preparation of arguments, called something like "argument-analysis". This idea is further supported by the fact that argument-analysis is needed anyway for reconstructing arguments in ordinary discourse as inferences, if only for the reason that arguments are usually not neatly structured in premises and conclusion. Given that we are ready to accept the relatively strong precondition that we formalize only inferences which are free of equivocation, the arguments considered give us no reason why we should not refer to utterances qua sentences in a theory of formalization. This proposal neatly fits established practice of formalization. It underlies logical analysis that is found in textbooks and philosophical discussions, even though it is rarely made explicit. Traces of this strategy can also clearly be found in Montague's groundbreaking work on formalization, where he proposes to use sentences of a disambiguated language as input for a procedure of formalization (Montague 1970).

If, however, we think that relying on argument-analysis undermines the traditional program of logic, we have to adopt a strategy for dealing with equivocation within a theory of formalization. Since equivocation is highly context dependent, such a theory can only be given if it is allowed to draw on information about the context of utterances. It therefore must deal with descriptions of utterances that include not only sentence-types, but also some information about the context of utterance. For this purpose a notion of "utterance-type" can be introduced and defined as, for example, a quadruple of sentence-type, speaker, place and time. Such a proposal has not been mentioned as an answer to (Q), since in this role it would again face the problem that one could not accommodate the fact that formulas must be able to substitute for utterances which are tokens of different sentence-types. But in the context of a theory of formalization, referring to utterance-types implies only that instances of different utterance-types must be treated in the same way by the theory. Again, this proposal can be found in an important paradigm for theories of formalization. It is basically what Davidson argues for when he insists that a theory of meaning should refer to utterances rather than sentences. (And he also argues that the adequacy of a 
formalization can only be judged in the context of a theory of meaning. Cf. Davidson 1980 , pp. 144-145, 1999, p. 35.) But he also concedes that it is an open question, which features of the situation of utterance turn out to be indispensable if we are to give such a theory (cf. Davidson 1984, pp. 8, 58, 149-150).

Assigning the task of handling equivocation to a theory of formalization implies that such a theory must include a great deal of syntactic, semantic and pragmatic analysis. This invites the objection that a theory of formalization should not address problems which are not logical, but linguistic in nature and therefore should be delegated to linguistics. To this one may reply (following Montague 1970) that there is no guarantee that linguistics will provide a theory of formalization with the information it needs, unless it is guided by logical considerations. If we put aside such worries about division of labour, we may propose that theories of formalization refer to utterances described as quadruples of sentence-type, syntactic, semantic and pragmatic structure. Grandy (1993, pp. 57-58), for example, puts forward such a solution under the label "fully specified strings", although not as an answer to (F) but to (Q). This strategy can be supported against utterance-types by another line of argument. Since a theory of formalization needs to be concerned exclusively with logical form, it need not bother identifying meanings or references of utterances or their parts, but only avoid the fallacy of equivocation. And for doing this, the structures included in fully specified strings, it is hoped, are sufficient; we do not need to know about speaker, time and so on. Davidson, on the other hand, must insist on including such information, because his theories of meaning are designed to give a systematic account of truth-conditions. That obviously involves more than representing logical forms, for not all truth-conditions are relevant to logical form. "Socrates is flying", for example, has the same logical form as "Theaitetos is flying" yet not the same truth-conditions.

All three proposals (sentences, utterance-types and fully specified strings) arguably are compatible with the traditional program of logic. What information, exactly, about utterances is needed for a theory of formalization is another question. Its answer depends on several factors. One is the distinction we want to draw between logical theory, preliminary analysis of argument and linguistic analysis. Others, such as the information needed to successfully address equivocation, are still subject to argument and research. Given that we do not yet have any reasonably comprehensive theories of formalization for an ordinary language, it may well be best to try alternative approaches. Nevertheless, something can be said about how (Q) could be answered, provided we had a theory of formalization.

\subsection{Some Possible Answers}

First of all, we may note that, together with a logical formalism, a theory of formalization would provide all that is needed for a complete theoretical account of the logical "core business" of proving validity. Giving a theory of valid inferences does not imply that one has to come up with an answer to the question of what the objects of logic are. Nevertheless, this question remains important owing to its significance for a philosophical understanding of logical practice, but also because 
of its connection with related questions about the objects of propositional attitudes and the bearers of truth and modality. I will therefore briefly examine some ideas how a theory of formalization could be used for answering (Q).

The most straightforward idea would be to claim that formulas stand for whatever we choose as an answer to (F). The three options considered in the preceding section (sentences, utterance-types and fully specified strings), however, are not viable candidates. They would just reintroduce some of the problems with sentences discussed in Sect. 2.3. Utterance-types and fully specified strings are ntuples including a sentence-type. Hence, they partition utterances into equivalence classes which are at least as fine-grained as those constituted by sentence-types and in fact are subclasses thereof. Consequently, (C4) could not be met if one of the last section's proposals for answering (F) was used as an answer to (Q).

We may also note that a theory of formalization would put us in a position to realize the utterance-and-content-strategy outlined in the second half of Sect. 2.1. Firstly, we can define the relation representing a logical form of an utterance appealed to in $(\mathrm{U})$ :

(32) $\phi$ represents a logical form of $\mathrm{u}$ iff $\phi$ is a formula and $\mathrm{u}$ is an utterance and there is an adequate formalization of $\mathrm{u}$ with $\phi$.

Secondly, a theory of formalization can be used to complete $\left(\mathrm{O}^{*}\right)$ since it must, at least implicitly, specify the conditions under which two utterances count as having the same content:

(33) For all utterances $\mathrm{u}_{1}$ and $\mathrm{u}_{2}: \mathrm{u}_{1}$ stands in relation $\mathrm{S}$ ("same content") to $\mathrm{u}_{2}$ iff there is a formalization $\Phi$ such that the sequence $\left\langle\mathrm{u}_{1}, \mathrm{u}_{2}\right\rangle$ can be adequately formalized by $\langle\Phi, \Phi\rangle$.

However, as we have seen in Sect. 2.1, the application of these principles is far too limited to provide a satisfactory account of the traditional program of logic.

More attractive ways of answering (Q) take up suggestions made in Sect. 2.4. First, a theory of formalization would make it possible to give an account of $\mathrm{X}$ and $\mathrm{R}$ by identifying $\mathrm{Xs}$ with literal verbalizations. $\mathrm{R}$ could then be defined as the relation that holds between an utterance and an $\mathrm{X}$ if and only if $\mathrm{X}$ is a literal verbalization of an adequate formalization of this utterance. To meet (C1), this account would again have to be relativized to the context of an inference. Spelling out (E), however, would no longer be necessary since equivocation will be dealt with in a theory of formalization. Such a solution may appear as a rather shallow technical manoeuvre not providing an informative answer to (Q). But this appearance, I think, is misleading. One advantage of this proposal is that it makes unmistakably clear the main finding of the preceding discussion: whatever the objects are formulas stand for, their relation to utterances must essentially be dependent on their logical forms. Furthermore, we may interpret literal verbalizations in their role as the objects of logic as an intermediate solution, for example, by taking them as standard expressions of propositions, which in turn have to be explained by some theory. This, of course, suggests that we could also bypass literal verbalizations and give an answer to (Q) by combining a theory of formalization with an account of propositions that takes propositions to be assigned to utterances 
on the basis of their logical forms. Such a solution could be developed for various theories of propositions. The crucial point concerns relation R. It can be framed as follows:

(P) $\mathrm{u}$ stands in relation $\mathrm{R}$ to $\mathrm{p}$ iff $\mathrm{u}$ is an utterance and $\mathrm{p}$ is a proposition and there is a formalization $\Phi$ such that (i) $\Phi$ is an adequate formalization of $u$ (according to the theory of formalization) and (ii) all utterances that can be adequately formalized by $\Phi$ express p (according to the theory of propositions).

As has been pointed out in Sect. 2.4, current theories of propositions are not designed for explaining (ii), but for the purpose of accounting for propositions as objects of propositional attitudes. Consequently, they focus on explaining the relationship between utterances and some structure more fine-grained than logical forms relevant for answering (Q). But if a theory of propositions lacks an explication of relation (ii), this does not imply that it is impossible to adapt it accordingly (see Salmon 1992 for a development in this direction). Such an account would certainly constitute a theoretically more ambitious solution than the ones considered above. Two challenges have to be met for working it out. A notion of expression which can be used for (ii) above has to be introduced and we need a theory of formalization.

In conclusion, I will present some retrospective comments on the question why the dispute about statements, propositions and sentences has not lead to a satisfactory answer to (Q) so far.

First, the debate has often addressed simultaneously (Q) and some different questions. For one thing, there is a certain danger of conflating (Q) and (F). For another, it is often assumed that $(\mathrm{Q})$ and the questions "What are the bearers of truth?" and "What do we relate to by propositional attitudes?" have one and the same answer. It would of course be desirable for reasons of systematicity that one could identify some kind of entity that does all these jobs. But as answers to (Q), many notions answering the latter questions either trivialize a great deal of logic or introduce logically irrelevant distinctions that block numerous logical proofs. In any case, it should not simply be taken for granted that all these questions admit for one common answer.

Secondly, attempts to answer (Q) have probably often followed a strategy of explaining what formulas stand for by appeal to some linguistic notion with more or less readily available criteria of identity. Expressing the same meaning, saying the same thing, being tokens of the same sentence-type and being identical qua utterance, even if not fully understood, are at least old acquaintances. Nevertheless, that these notions are readily available and seemingly unproblematic should not deceive anybody about their unsuitability for answering (Q).

Thirdly, proposals informed by logic, such as logically structured propositions, tend to overlook that $(\mathrm{Q})$ does not only call for specifying a class of objects, but also addresses the relationship between formulas and ordinary language. Moreover, it does not suffice to claim that logical forms are a crucial factor in determining what propositions utterances express. A theoretical account of this relationship is needed for an informative answer to (Q), and that calls for a theory of formalization, just as 
logical proofs of valid inferences do. If most advocates of propositions neglect this, they are in good company. But that is no reason for giving up this part of the traditional program of logic.

Acknowledgements This paper thoroughly revises the points I made in Brun (2004, Chap. 5). Earlier versions have been presented in Bern, Hanover, Lund and Neuchâtel. For discussions and feedback, I am indebted to Michael Baumgartner, Jean-Yves Béziau, Richard Grandy, Paul Hoyningen-Huene, Timm Lampert and Klaus Petrus. Special thanks go to Dominique Kuenzle for collaboration on one of the very first drafts and to two anonymous referees of this journal for extremely helpful comments.

\section{References}

Bealer, G. (1993). A solution to Frege's puzzle. Philosophical Perspectives, 7, 17-60.

Blau, U. (1977). Die dreiwertige Logik der Sprache: Ihre Syntax, Semantik und Anwendung in der Sprachanalyse. Berlin: de Gruyter.

Brandom, R. B. (1994). Making it explicit: Reasoning, representing and discursive commitment. Cambridge, MA: Harvard University Press.

Brun, G. (2004). Die richtige Formel: Philosophische Probleme der logischen Formalisierung (2nd ed.). Frankfurt a. M: Ontos.

Carnap, R. (1956). Meaning and necessity: A study in semantics and modal logic (2nd ed.). Chicago: University of Chicago Press.

Chomsky, N. (1986). Knowledge of language: Its nature, origin, and use. Westport: Praeger.

Chomsky, N. (1995). The minimalist program. Cambridge, MA: MIT Press.

Collins, J. (2003). Expressions, sentences, propositions. Erkenntnis, 59, 233-262.

Cresswell, M. J. (1985). Structured meanings: The semantics of propositional attitudes. Cambridge, MA: MIT Press.

Davidson, D. (1963). The method of extension and intension. In P. A. Schilpp (Ed.), The philosophy of Rudolf Carnap (pp. 311-349). La Salle: Open Court.

Davidson, D. (1980). The logical form of action sentences. Criticism, comment, and defence. In Essays on actions and events (pp. 105-148). Oxford: Oxford University Press.

Davidson, D. (1984). Inquiries into truth and interpretation. Oxford: Oxford University Press.

Davidson, D. (1990). The structure and content of truth. Journal of Philosophy, 87, 279-328.

Davidson, D. (1999). Intellectual autobiography. In L. E. Hahn \& P. A. Schilpp (Eds.), The philosophy of Donald Davidson (pp. 1-70). Chicago: Open Court.

Epstein, R. L. (2001). The semantic foundations of logic: Predicate logic. Belmont: Wadsworth.

Frege, G. [1879] (1988). Begriffsschrift, eine der arithmetischen nachgebildete Formelsprache des reinen Denkens. In Begriffsschrift und andere Aufsätze. Darmstadt: Wissenschaftliche Buchgesellschaft. Translated in van Heijenoort, J. (Ed.). (1967). From Frege to Gödel: A source book in mathematical logic, 1879-1931 (pp. 1-82). Cambridge, MA: Harvard University Press (References are to the original pagination).

Frege, G. [1918] (1967). Der Gedanke: Eine logische Untersuchung. In Kleine Schriften (pp. 342-362). Darmstadt: Wissenschaftliche Buchgesellschaft. Translated in Beaney, M. (Ed.). (1997). The Frege reader (pp. 325-345). Oxford: Blackwell (References are to the original pagination).

Frege, G. (1969). Nachgelassene Schriften, Hamburg: Meiner. Translated as Frege, G. (1979). Posthumous writings. Oxford: Basil Blackwell (References are to the German/English edition).

Goodman, N. (1976). Languages of art: An approach to a theory of symbols (2nd ed.). Indianapolis: Hackett.

Grandy, R. E. (1993). What do "Q" and "R" stand for anyway? In R. I. G. Hughes (Ed.), A philosophical companion to first-order logic (pp. 50-61). Indianapolis: Hackett.

Higginbotham, J. (1991). Belief and logical form. Mind and Language, 6, 344-369.

Hoyningen-Huene, P. (2004). Formal logic: A philosophical approach. Pittsburgh: University of Pittsburgh Press. Translation of Hoyningen-Huene, P. (1998). Formale Logik: Eine philosophische Einführung. Stuttgart: Reclam.

Iacona, A. (2002). Propositions. Genova: Name. 
Kalish, D., Montague, R., \& Mar, G. (1980). Logic: Techniques of formal reasoning (2nd ed.). Fort Worth: Harcourt, Brace and Jovanovich.

Kaplan, D. (1989). Demonstratives. An essay on the semantics, logic, metaphysics, and epistemology of demonstratives and other indexicals. In J. Almog, J. Perry \& H. Wettstein (Eds.), Themes from Kaplan (pp. 481-563). Oxford: Oxford University Press.

Kaplan, D. (1990). Words. Proceedings of the Aristotelian Societysupplement, 64, 93-119.

King, J. C. (1995). Structured propositions and complex predicates. Noûs, 29, 516-535.

King, J. C. (1996). Structured propositions and sentence structure. Journal of Philosophical Logic, 25, 495-521.

King, J. C. (2005). Structured propositions. In E. N. Zalta (Ed.), The Stanford Encyclopedia of Philosophy. http://plato.stanford.edu/archives/sum2005/entries/propositions-structured/.

Lampert, T. (2005). Klassische Logik: Einführung mit interaktiven Übungen (2nd ed.). Frankfurt a. M.: Ontos.

Larson, R. K., \& Ludlow, P. (1993). Interpreted logical forms. Synthese, 95, 305-355.

Larson, R. K., \& Segal, G. (1995). Knowledge of meaning: An introduction to semantic theory. Cambridge, MA: MIT Press.

Lemmon, E. J. (1987). Beginning logic (2nd ed.). London: Chapman and Hall.

LePore, E. (2000). Meaning and argument: An introduction to logic through language. Malden: Blackwell.

LePore, E., \& Ludwig, K. (2007). Donald Davidson's truth-theoretic semantics. Oxford: Clarendon Press.

Link, G. (1979). Montague-Grammatik. München: Fink.

Martin-Löf, P. (1996). On the meanings of the logical constants and the justifications of the logical laws. Nordic Journal of Philosophical Logic, 1, 11-60. http://www.hf.uio.no/ifikk/filosofi/njpl/vol1no1/ meaning/meaning.html. Accessed on 16 April 2008.

Menzel, C. (1993). The proper treatment of predication in fine-grained intensional logic. Philosophical Perspectives, 7, 61-87.

Montague, R. [1970] (1974). Universal grammar. In Formal philosophy: Selected papers (pp. 222-246). New Haven: Yale University Press.

Nolan, R. (1969). Truth and sentences. Mind, 78, 501-511.

Peirce, C. S. (1902). Logic. In J. M. Baldwin (Ed.), Dictionary of philosophy and psychology (Vol. 2, pp. 20-23). New York: MacMillan.

Penco, C. (2003). Frege: Two theses, two senses. History and Philosophy of Logic, 24, 87-109.

Perry, J. (1979). The problem of the essential indexical. Noûs, 13, 3-21.

Quine, W. V. O. (1960). Word and object. Cambridge, MA: MIT Press.

Quine, W. V. O. (1982). Methods of logic (4th ed.). Cambridge, MA: Harvard University Press.

Ramsey, F. P. [1925] (1990). Universals. In Philosophical papers (pp. 8-30). Cambridge: Cambridge University Press.

Richard, M. (1990). Propositional attitudes: An essay on thoughts and how we ascribe them. Cambridge: Cambridge University Press.

Rosenberg, J. F. (1986). Die Autorität der logischen Analyse: Einige Provokationen. Neue Hefte für Philosophie, 26, 69-88.

Sainsbury, M. (2001). Logical forms: An introduction to philosophical logic (2nd ed.). Oxford: Blackwell. Salmon, N. (1992). On content. Mind, 101, 733-751.

Soames, S. (1987). Direct reference, propositional attitudes, and semantic content. Philosophical Topics, 15, 47-87.

Stalnaker, R. (1976). Propositions. In A. F. MacKay \& D. D. Merrill (Eds.), Issues in the philosophy of language: Proceedings of the 1972 Oberlin Colloquium in Philosophy (pp. 79-91). New Haven: Yale University Press.

Stalnaker, R. (1984). Inquiry. Cambridge, MA: MIT Press.

Strawson, P. F. (1952). Introduction to logical theory. London: Methuen.

Wittgenstein, L. (1922). Tractatus Logico-Philosophicus. London: Routledge and Kegan Paul.

Zalta, E. N. (1988). Intensional logic and the metaphysics of intentionality. Cambridge, MA: MIT Press. 